Résumés des conférences et travaux

\title{
Liste des doctorats EPHE soutenus à la Section pendant l'année 2007-2008
}

par ordre alphabétique des noms d'auteur

\section{QpenEdition}

\section{Journals}

Édition électronique

URL : https://journals.openedition.org/ashp/599

DOI : 10.4000/ashp.599

ISSN : 1969-6310

Éditeur

Publications de l'École Pratique des Hautes Études

Édition imprimée

Date de publication : 1 octobre 2009

Pagination : xii-xiv

ISSN : 0766-0677

Référence électronique

"Liste des doctorats EPHE soutenus à la Section pendant l'année 2007-2008 », Annuaire de l'École pratique des hautes études (EPHE), Section des sciences historiques et philologiques [En ligne], 140 | 2009, mis en ligne le 23 octobre 2009, consulté le 06 juillet 2021. URL : http://journals.openedition.org/ashp/ 599 ; DOI : https://doi.org/10.4000/ashp.599

Ce document a été généré automatiquement le 6 juillet 2021.

Tous droits réservés : EPHE 


\section{Liste des doctorats EPHE soutenus à la Section pendant l'année 2007-2008}

par ordre alphabétique des noms d'auteur

1 L'Ayâdgâr î Jamâspig : un testo escatologico zoroastriano. Edizione e commentario dell'opera, par Domenico AgostinI, sous la direction de M. Philip HUYSE (EPHE) et de M. Carlo CERETI (université La Sapienza), le 14 février 2008.

2 Le génie militaire de Khaled Ibn Al-Walid, par Mohamed AL-SuWAIDI, sous la direction de M. François DÉROCHE, le 15 décembre 2007.

3 Le milieu intellectuel juif en Italie au XVI eiècle : les manuscrits de Raphaël Salomon ben Jacob Ha-Cohen de Prato, par Élodie ATTIA, sous la direction de $\mathrm{M}^{\text {me }}$ Judith OLSZOWY-SCHLANGER, le 7 avril 2008.

$4 \quad$ L'influence de la représentation du monde germanique sur la pensée, l'action et la production littéraire de Charles de Gaulle - 1890-1949, par Philippe BEDOURET, sous la direction de M. Jacques LE RIDER, le 17 juin 2008.

5 Les monuments funéraires de Saint-Denis à l'époque moderne, par Julian BLuNK, sous la direction de M. Guy-Michel LeProux (EPHE) et de M. Bruno KLeIN (université technique de Dresde), le 30 janvier 2008.

6 La médecine arabe dans les universités italiennes : étude des commentaires au Canon d'Avicenne en Italie au XIV ${ }^{e}$ siècle, par Joël CHANDELIER, sous la direction de $\mathrm{M}^{\text {me }}$ Danielle JACQUART, le 8 décembre 2007.

7 Étude comparative des embarcations de pêche et de cabotage en Méditerranée occidentale.

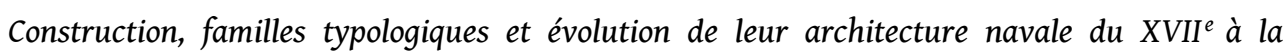
première moitié $\mathrm{du} \mathrm{XX}^{e}$ siècle, par José-Luis CORTES, sous la direction de M. Jean-Francois BELHOSTE, le 24 mai 2008.

8 Recherches sur l'histoire du texte du De materia medica de Dioscoride, par Marie CRONIER, sous la direction de $\mathrm{M}^{\mathrm{me}}$ Brigitte MondRAIN, le $1^{\text {er }}$ décembre 2007. 
9 Le supplétisme dans les formes de gradation en grec ancien et dans les langues indoeuropéennes, par Éric DIEU, sous la direction de M. Charles DE LAMBERTERIE, le 8 décembre 2007.

Les villages et les sociétés villageoises en Ifriqiya au haut Moyen Âge (II-V ${ }^{e}$ s. H. / VIII ${ }^{e}-\mathrm{XI}^{e}$ s. apr. J.-C.). Approche historique et archéologique, par Lamjed DRIDI, sous la direction de M. Michel TERRASSE, le 12 juin 2008.

11 Clausewitz et la réflexion sur la guerre en France, 1807-2007, par Benoît DURIEUX, sous la direction de M. Hervé COUTAU-BÉGARIE, le 6 décembre 2007. La syllabe copte : de la philologie à la phonologie, par Jean-Louis FORT, sous la direction de M. Gérard ROQUET, le 15 décembre 2007. La constitution de la marine de guerre hellénique et la force navale de la Grèce (1900-1913), par Panagiotos FourAKIS, sous la direction de M. Hervé COUTAU-BÉGARIE, le 21 février 2008. Les verbes de déplacement dans les textes des sarcophages, par Carlos GRACIA ZAMACONA, sous la direction de M. Pascal Vernus, le 5 avril 2008. Thomas Mann et Marcel Proust - Tempus multiformum, étude comparatiste, par RalucaMihaela HeRgheligiu, sous la direction de M. Jacques Le RIDER, le 28 mai 2008. Le Dieu celtique Lugus, par Gaël HiLy, sous la direction de M. Pierre-Yves LAMBERT, le $1^{\text {er }}$ décembre 2007.

17 La construction de la déviance des mercenaires dans le XVI et le XVII siècle, par Jan Willem HUNTERBRINKER, sous la direction de M. Frédéric BARBIER (EPHE) et de M.Gerd SCHWERHOFF (université technique de Dresde), le 30 janvier 2008.

Les receveurs municipaux sous la III République, par Catherine JUMEAU, sous la direction de M. François MONNIER, le 18 décembre 2007.

19 L'organisation militaire de Philippe de Flandre dit « le Bon » dans les grands Pays-Bas de 1419 à 1467, par Guillaume LEVECQ, sous la direction de M. Hervé COUTAU-BÉGARIE, le 22 février 2008.

20 L'écrit, le livre et la publicité. Les engagements d'un aristocrate éclairé de Bohême : Franz Anton Hartig (1758-1797), par Claire MADL, sous la direction de M. Frédéric BARBIER, le 26 novembre 2007.

21 La pratique de l'auto-représentation architecturale à partir de l'exemple des cultures métropolitaines de Paris et Berlin, par Christian PETERS, sous la direction de M. Jean-Michel LENIAUD (EPHE) et de M. Karl-Siegbert REHBERG (université technique de Dresde), le 21 janvier 2008.

22 Entretenir un monument gothique sous l'Ancien Régime: la Sainte-Chapelle du Palais, par Magalie QUINTARD, sous la direction de M. Jean-Michel LENIAUD, le 3 décembre 2007.

23 Éthique et réflexion militaire française dans la première moitié du XVII ${ }^{e}$ siècle, par Olivier RIBIÈRE, sous la direction de M. Hervé COUTAU-BÉGARIE, le 7 mars 2008.

L'Église de France et les emblèmes de ses évêques vers 1645-vers 1920. Héraldique, sigillographie, numismatique, paramentique, prosopographie des évêques en France et en quelques territoires adjacents, par Christophe RousSEAU LEFEBVRE, sous la direction de M. Michel PASTOUREAU, le 25 janvier 2008. 
La participation des femmes au journalisme politique et littéraire. Italie et France entre XIXe et $\mathrm{XX}^{e}$ siècle, par Maria Cecilia VIGNuZZI, sous la direction de M. Gilles PÉcout (EPHE) et de $\mathrm{M}^{\mathrm{me}}$ Ilaria Porciani (université de Bologne), le 23 juin 2008.

Le palais des Papes de la Révolution à nos jours, par Dominique VINGTAIN, sous la direction de M. Jean-Michel LeNIAUD, le 8 janvier 2008.

27 Contribution à une recherche d'éléments Môn dans l'épigraphie khmère de la période préangkorienne, par Daoruang WitTAYARAT, sous la direction de M. Pierre-Sylvain FilLIOZAT, le 10 janvier 2008.

\section{INDEX}

Thèmes : Doctorats soutenus à la Section 